\title{
Demografia informal: cómo utilizar las redes sociales para construir una muestra etnográfica sistemática de mujeres mexicanas en ambos lados de la frontera*
}

\author{
Jennifer S. Hirsch** \\ Constance A. Nathanson**
}

En este articulo se discutirá cómo el muestreo etnográfico sistemático puede fortalecer la posibilidad de hacer una generalización etnográfica y ayudar a promover la comunicación interdisciplinaria. El estudio al cual se réfiere este trabajo examina las diferencias en las prácticas sexuales y de salud reproductiva, asi como las ideas de dos grupos de mujeres provenientes de la misma área en México: la primera generación de inmigrantes en Atlanta, y sus hermanas y amigas que permanecen en la comunidad de expulsión en el occidente de México.

Se comenzará con la exposición de un estudio etnográfico sobre la sexualidad que ha utilizado este método de muestreo, para argumentarien la siguiente sección por quéel muestreo fue particularmente importante en este contexto especifico de investigación. En la siguiente sección se revisan algunas de las diferencias teóricas (y epistemológicas sub. yacentes) entre la manera como los demógrafos y los antropólogos eligen a los sujetos para ser entrevistados. Finalmente, la última sección comprende la construcción de una muestra etnográfica sistemática, incluyendo la descripción de algunas dificultades enfrentadas durante el proceso.

\section{Preguntas de investigación y revisión de métodos}

Este estudio examina las diferencias en las ideas y las prácticas sexuales y de salud reproductiva entre dos grupos de mujeres provenientes de la misma localidad en México: unas son inmigrantes de primera generación en Atlanta y las otras son sus hermanas y amigas aún en la comunidad de origen. La atención se centra en las concepciones de las mujeres mexicanas sobre el modo en que el con-

* El trabajo de campo realizado para este estudio fue auspiciado por el National Science Foundation Program in Cultural Antropology (\#SBR-9510069), y por la Johns Hopkins School of Hygiene and Public Health. El análisis de la investigación se llevó a cabo gracias a una donación del International Migration Program at the Social Research Council. Además, la ayuda de muchas otras personas fue invaluable para el desarrollo y la realización de ese proyecto, incluyendo a Arthur Murphy del departamento de Antropología de la Univesidad Estatal de Georgia, a la hermana Barbara y su equipo de trabajo del Mercy Mobile Health Care de Atlanta, al cónsul mexicano en Atlanta, Teodoro Maus, a Ivonne Szasz y todos los participantes en el Seminario sobre Sexualidad y Género de El Colegio de México cuyos comentarios sobre mi propuesta original fueron generosos y, finalmente, a las propias mujeres que tan amablemente me invitaron a sus casas y a sus vidas.

** Johns Hopkins University. 
texto social donde viven afecta sus relaciones sexuales y reproductivas. El estudio aborda tres preguntas: $i$ ) ¿cómo difieren las construcciones de género y sexualidad de estas dos comunidades?; ii) ¿cómo difieren los recursos, estrategias y metas de las mujeres, tanto entre los grupos como dentro de cada uno?; iii) ¿qué consecuencias tiene cada grupo de diferencias en el uso que hacen las mujeres de los métodos para controlar la natalidad y prevenir las enfermedades?

La obtención principal de datos se conforma con las historias de vida dirigidas de 13 mujeres en Atlanta y trece mujeres en México (Mintz, 1960; Abu-Lughod, 1993; Gulati, 1981). Para situar estas historias de vida en un contexto más amplio, otras formas de recolección de datos incluyen entrevistas no estructuradas con amistades y parientes de las informantes clave, dibujos de las historias de vida, ${ }^{1}$ mapas corporales ${ }^{2}$ y observación participativa. El trabajo de campo comenzó en agosto de 1995 y concluyó en febrero de 1997.

Además de apoyarse en investigación migratoria reciente sobre inmigrantes mexicanos que viven en comunidades transnacionales (Rouse, 1991, 1992; Kearney, 1986; Massey et al., 1993), el estudio está arraigado en dos áreas teóricas. Una breve revisión de cada área indicará las razones iniciales para considerar el muestreo. El primer concepto fundamental es la idea de que la sexualidad se construye socialmente. En palabras de Carole Vanee, los construccionistas sociales conciben que "actos sexuales físicamente idénticos pueden variar en significancia y sentidos subjetivos dependiendo de cómo se definan y comprendan en distintas culturas" (Vanee, 1991: 878). Esto implica que dentro de una cultura, los sentidos asociados a conductas y relaciones específicas serán compartidos por los miembros de dicha cultura. Por lo tanto, no es necesario hablar con cada persona, y ni siquiera con una muestra probabilística representativa, para obtener

\footnotetext{
${ }^{1}$ Antes de comenzar las entrevistas de la historia de vida, se proporciona a las mujeres marcadores de colores y una hoja grande de papel y se les pide que dibujen los momentos más importantes de sus vidas, tanto del pasado como del presente y futuro. Los dibujos constituyen una forma más participativa y menos restringida de que las mujeres definan los momentos críticos de sus historias vitales, así como de que exploren aspectos personales en relación con la comunidad.

${ }^{2}$ Los mapas corporales son otro método de investigación participativa (Cornwall, 1992). Antes de comenzar la cuarta de las seis entrevistas sobre la historia vital, se pide a las mujeres que rellenen una silueta de un cuerpo femenino con sus ideas sobre lo que hay dentro de una mujer y dónde crecen los bebés. La técnica es útil para averiguar los variados modelos etnomédicos de reproducción de las mujeres.
} 
una imagen coherente de la construcción de la sexualidad en un grupo particular. Por el contrario, la meta sería hablar con menos personas, pero lo suficientemente a fondo como para obtener los detalles de este complejo atributo compartido.

Aunque se pueda hablar con relativamente pocas personas, es importante que sean seleccionadas propositivamente. La ideología de género, la ideología religiosa y las cuestiones de sexualidad e identidad nacional son influencias compartidas en la sexualidad, pero cualquier recuento de una sexualidad socialmente construida es estático a menos que también aborde cuestiones de poder y recursos. En otras palabras, lo que la gente de hecho hace con una ideología de la sexualidad variará tanto por sus historias individuales como por sus posiciones sociales, por los roles y elecciones a que tiene acceso. Puede dominar una cierta construcción social compartida de la sexualidad, aunque es probable que en virtud del nacimiento, dinero o sexo algunos tengan un rango más amplio de acción que otros. Además, dentro del rango de elecciones disponible, puede haber una variedad de maneras en que los individuos usen esta ideología para lograr sus propios fines. Todo esto sugiere que los informantes deben seleccionarse propositivamente para maximizar la variación en la posición social y las experiencias vitales.

La otra ancla teórica del estudio es una adaptación crítica de la teoría del regateo. Esta teoría sugiere que la reproducción y la sexualidad juegan un papel en la administración estratégica que hacen las mujeres de sus recursos para alcanzar metas determinadas culturalmente. Este estudio explora dos aspectos de la teoría del regateo. Primero, ¿̨hasta qué punto las mujeres mexicanas de dos contextos sociales conciben su sexualidad o fecundidad como algo que pueden administrar? En otras palabras, ¿¿de qué formas y en qué situaciones son estas mujeres sujetos (y no objetos) sexuales que comprenden sus cuerpos como propiedad individual (y no colectiva)? Segundo, en caso de que sí administren de manera estratégica su sexualidad, ¿cuáles son sus metas y cómo moldean estas metas sus prácticas de prevención de enfermedades de transmisión sexual (ETS) y control de la fecundidad? La teoría del regateo integra influencias materiales (acceso a una variedad de recursos determinados por la cultura) e ideológicas (como las ideologías de género). Por lo tanto, para probar que la teoría del regateo sea aplicable en el contexto de un país en desarrollo, la investigación debe incluir una muestra que 
sea heterogénea tanto en términos de ideología como de acceso a los recursos. ${ }^{3}$

¿Por qué considerar un muestreo para un estudio etnográfico sobre sexualidad y salud reproductiva entre mujeres mexicanas en ambos lados de la frontera?

Como se esbozó antes, las bases teóricas del estudio sugieren no sólo varias razones para pensar en un muestreo, sino también parámetros específicos, como son una muestra relativamente reducida y diversidad social dentro del grupo pequeño. Éstos se mantendrían, no obstante, para cualquier estudio etnográfico sobre sexualidad que utilice el enfoque teórico descrito. Hay otras dos razones por las cuales es imperativo considerar un muestreo en un estudio sobre mujeres mexicanas en ambos lados de la frontera. Primero, como se ha señalado con frecuencia en los estudios demográficos sobre inmigración en la frontera México-Estados Unidos, no existe un marco de muestreo evidente. Como no hay un marco muestral fácilmente disponible, no hay por lo tanto una manera directa de saber en qué formas el grupo pequeño y socialmente diversificado que se seleccione pueda diferir o asemejarse a la comunidad mayor de inmigrantes mexicanos en Atlanta o a los mexicanos en el oeste de México que tienen parientes en Estados Unidos.

Tampoco fue posible la solución etnográfica por excelencia al problema de cómo localizar -tanto logística como sociológicamenteun pequeño grupo de informantes dentro de su localidad. A diferencia de estudios sobre pueblos pequeños, en la comunidad de inmigrantes mexicanos no hay ni fronteras visibles ni padrones de miembros claramente definidos. En Atlanta hay demasiados mexicanos como para que todos se conozcan y están demasiado dispersos como para que el estudio se haya podido concentrar en un área sin averiguar más sobre por qué las personas eligieron vivir donde lo hicieron (mapa 1). Así, es importante comprender que esta cuestión del muestreo no es sólo para complacer al público demográfico. En estudios

${ }^{3}$ Agradezco a los miembros del Seminario sobre Sexualidad y Género del Programa Salud Reproductiva y Sociedad de El Colegio de México por alentarme a asumir una perspectiva crítica mayor respecto a la teoría del regateo, y por señalar algunos caminos que permiten un enfoque cultural mayor. 
MAPA 1

Estimaciones de la población de origen mexicano en Adanta por condado, 1995

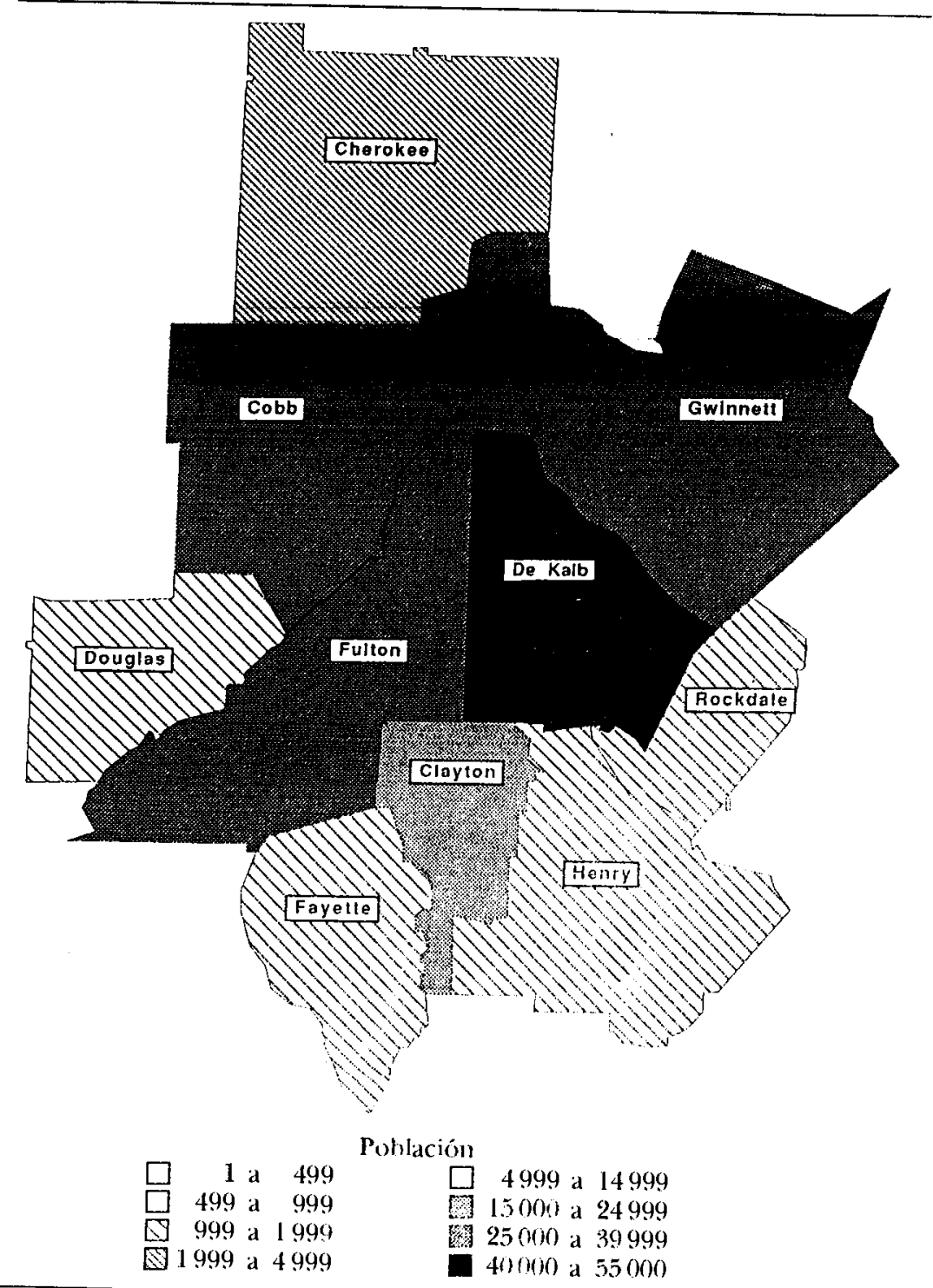

Nota: Realizado por el Centro de Investigación Aplicada en Antropología de la Universidad Estatal de Georgia. 
etnográficos en áreas urbanas complejas, los investigadores deben luchar con la idea de qué significa buscar una comunidad en un área urbana, así como con la pregunta de en qué formas las personas seleccionadas como informantes son parecidas o distintas de los otros miembros de la comunidad.

En encuestas de población en un país, estado o ciudad, los investigadores pueden construir una muestra a partir de mapas censales, que supuestamente abarcan a la población completa. Como este estudio cubre la frontera, no hay un marco de muestreo que por sí solo abarque toda la zona de estudio, ni es probable que haya indicadores en los censos de ambos países que permitan relacionar sus datos. Tampoco hubiera sido posible elegir casos del censo de Estados Unidos y rastrearlos etnográficamente al otro lado de la frontera. Aunque en el censo de 1990 hubieran aparecido como de origen mexicano, podrían haberse mudado de localidad o regresado a México. Además, como una proporción considerable de la comunidad mexicana de Atlanta ha excedido el límite de una visa de turista o está en Estados Unidos sin permiso oficial (lo que el ins llama "entrada sin inspección"), es poco probable que la comunidad haya sido de hecho enumerada en 1990. Las organizaciones comunitarias sugieren que el censo de 1990 omitió casi a la mitad de los mexicanos que estaban en Atlanta en aquel tiempo. ${ }^{4}$ En todo caso, en el censo de 1990 se preguntó a los extranjeros residentes el país de nacimiento, pero no el estado o ciudad de residencia original en dicho país.

La segunda razón por la cual es importante tratar con cuestiones de muestreo es que este estudio seguramente tendrá implicaciones en la elaboración de las políticas. La fecundidad de las mujeres mexicanas en Estados Unidos es mucho menor que la de sus pares en México (Kahn, 1988). Esta reducción puede ocurrir en gran medida a pesar (y no como consecuencia) de la disponibilidad y costo de los servicios de planificación familiar en Estados Unidos, aunque el creciente número de mujeres mexicanas seguirá necesitando esos servicios. Si los servicios se extienden para proteger y promover la salud reproductiva de estas mujeres, un buen punto de partida podría ser conocer qué creen realmente que es la salud reproductiva. Por otro lado, el porcentaje de casos de sida en áreas rurales de México aumentó a 4\% en 1993,

\footnotetext{
${ }^{4}$ Chuck Johnson, Asociación Latinoamericana, Atlanta, Georgia, octubre de 1994, comunicación personal; Arthur Murphy, Centro de Investigación Aplicada en Antropología, Atlanta, Georgia, octubre de 1994, comunicación personal.
} 
de 0\% en 1983 (Bronfman y Gómez Dantes, s.f.). Los funcionarios de salud pública especulan que bien podría haber una conexión con los flujos migratorios, y el gobierno mexicano ha respondido con una campaña radiofónica para educar a los hombres mexicanos en Estados Unidos sobre la importancia de usar preservativo con parejas secundarias. No existen datos demográficos sobre las tasas de ETs entre las mujeres en México, aunque los datos etnográficos sugieren que las mujeres con frecuencia se ven forzadas a reconocer la infidelidad de sus esposos porque en sus visitas anuales les traen "una infección". Aquí caben sin duda los esfuerzos binacionales de prevención de ETs, y un estudio como éste, que rastrea los perfiles de la sexualidad en ambos lados de la frontera, es una base importante para construir tal campaña.

Si los resultados del estudio van a ser accesibles a un público interdisciplinario que puede estar más acostumbrado a los resultados de encuestas que a la etnografia, un primer paso importante es tener un plan para elegir a los entrevistados, es decir, un plan de muestreo. Las técnicas de muestreo probabilístico de los demógrafos son, por lo menos en parte, lo que les ha dado el monopolio en la producción de verdades para el consumo de quienes elaboran las políticas. Como en Estados Unidos los que elaboran políticas justifican cada vez más sus respuestas a las cuestiones públicas sugiriendo que simplemente están respondiendo a los datos (Asad, 1994), se vuelve más importante que los antropólogos que quieren tener voz en la elaboración de las políticas sean transparentes en cuanto a la manera en que llegaron a sus conclusiones. Se verá más adelante cómo la generalización etnográfica se distingue de la generalización estadística -lo importante por el momento es que para que la etnografía sea útil para formar políticas, debe ser generalizable, y para ser generalizable debe presentar y seguir un plan de muestreo. Las historias de vida de un número relativamente reducido de mujeres mexicanas pueden ser retratos ricamente evocativos de cómo algunas mujeres han respondido a las restricciones y oportunidades en sus vidas al tomar ciertas decisiones sobre sexualidad y reproducción. Sin embargo, hablar sólo de la riqueza de los datos pasa por alto la forma en que estas historias se relacionan con la comunidad más amplia de mujeres mexicanas en Atlanta o en su ciudad de origen. Además de conseguir nna opinión con autoridad por medio de un análisis coherente del trabajo y notas de campo, los etnógrafos $-y$ en particular aquellos que quieren que su trabajo traspase las fronteras disciplinarias- deben explicar exactamente por qué sus hallazgos son generalizables. A continuación se presentan la 
teoría y práctica para la construcción de una muestra etnográfica sistemática.

Diferencias disciplinarias en los principios de muestreo

A pesar del interés generalizado por hacer investigación interdisciplinaria sobre sexualidad, el equivalente etnográfico de lo que los encuestadores llaman muestreo no ha recibido mucha atención. Una teoría del muestreo es fundamentalmente una epistemología, una teoría sobre cómo aprender algo confiable y válido sobre un grupo completo hablando sólo con un cierto número de sus miembros. Para poder extrapolar al grupo completo la información obtenida del número reducido de individuos, debe utilizarse una teoría sobre la relación entre el grupo y los miembros individuales. Debe aclararse aquí cómo las tradiciones intelectuales de la etnografia y la demografia tienen suposiciones distintas acerca de dicha relación.

Se pueden comparar varios principios generales entre los muestreos etnográfico y de encuestas (cuadro 1). El muestreo demográfico es universalizador y orientado hacia el individuo. Al construir una muestra para una encuesta demográfica, se comienza con ciertas categorías a priori, como raza o etnicidad, sexo y edad. Para los fines de una encuesta, la mujer blanca de 35 años de edad, casada, de clase media y con tres hijos, elegida para la entrevista, es tan adecuada como cualquier otra mujer de 35 años de la misma raza, ingresos, número de hijos y estado civil. Rara vez se reconoce la organización social que existe como nivel intermedio entre el individuo y la población. En una encuesta demográfica basada en los hogares, por ejemplo, la muestra consiste en un individuo de cada hogar seleccionado. Esto quiere decir que un estadístico con mapas censales recientes puede definir un marco muestral para un país que nunca ha visitado. Es sólo ignorando los dédalos de la organización social y tomando individuos autónomos como unidades de muestreo que los encuestadores pueden:justificar el uso de las mismas técnicas de muestreo en todo el mundo.

Donde los demógrafos son universales, los etnógrafos son particulares en cuanto al enfoque, concentrándose sobre todo en la organización social. En la etnografía el método de muestreo que de hecho se utilice dependerá tanto de un conocimiento razonable de la sociedad estudiada como del tema de estudio. En el caso de los inmi- 
CUADRO 1

Principios de muestreo

\begin{tabular}{ll}
\hline Etnografía & Investigación por encuestas \\
\hline Particularistas & Universalista \\
Estructurado (propositivo) & Aleatorio \\
Profundo (intensivo) & Amplio (extensivo) \\
Representativo de tipos & Representativo de la distribución \\
Personal & Anónimo \\
\hline
\end{tabular}

grantes mexicanos, las principales redes sociales son regionales y familiares, basadas en las comunidades de origen en México (Hondagneu-Sotelo, 1994; Massey et al., 1987; Mummert, 1992; Fernández, 1988; Trigueros y Rodríguez Piña, 1988; Alarcón, 1988). El foco es precisamente el nivel de organización social que en general resulta invisible para la investigación por encuestas orientada hacia el individuo -la idea es estudiar a la gente dentro de las redes sociales, y no despajada de ellas. Tanto la investigación previa como el marco teórico sugerirán posibles categorías clave de informantes, aunque éstas deben confirmarse con el trabajo de campo inicial.

En tercer lugar, se entrevista a relativamente pocos informantes varias veces y a fondo. Siguiendo los principios etnográficos de cómo se conoce algo sobre un grupo de personas -la epistemología etnográfica, pues- no es necesario tener enormes muestras. La posibilidad de generalizar los datos de las encuestas deriva de usar muestras suficientemente grandes como para interpretar los datos de modo que con un cierto nivel de confianza representen a toda la población. Datos de encuestas estructuradas de trece mujeres en México y trece en Estados Unidos serían inútiles en términos de generalización estadística. La información recabada con una muestra tal necesariamente manejaría sólo de manera superficial conductas y actitudes, y la falta de profundidad no se compensaría con un aumento equivalente en la extensión.

La generalización etnográfica, sin embargo, es un concepto distinto. No trata con la distribución de las conductas, sino con su significado; la idea fundamental es que la cultura es un atributo grupal, no una característica individual. Las mujeres dicen cosas diferentes sobre qué constituye un buen matrimonio o qué tipos de actividad sexual pueden ocurrir dentro de un noviazgo "decente", aunque esto que comparten con el etnógrafo no es una invención individual. Afir- 
mar que la cultura es un atributo de grupo significa que escuchando a suficientes mujeres durante un tiempo considerable, haciendo buenas preguntas y siendo un observador perceptivo, un etnógrafo puede desarrollar una imagen que sea internamente coherente de los significados que subyacen en ciertas acciones. Los relatos de las mujeres son producto de sus historias individuales, su clase social y su cultura compartida.

Un estudio de este tipo no es útil para descubrir qué porcentaje de mujeres utiliza el coitus intermuptus como control de la natalidad. Es el único modo, sin embargo, de saber que muchas mujeres recién casadas prefieren el riesgo de embarazarse al riesgo emocional percibido que representaría para su matrimonio usar un método más efectivo. Estas mujeres parecen sentir que su fecundidad potencial es su recurso más valioso dentro de la relación, así que si van a demorarse en tener hijos quieren ver que sus parejas demuestren su compromiso participando activamente en el control de la natalidad. De manera similar, al final del estudio no será posible concluir cuántas de las muijeres son más o menos poderosas que sus maridos -de hecho, aquí la mera idea de dividir el poder en una variable categórica es un anatema. Sin embargo, será posible afirmar con confianza que tener hijos nacidos en Estados Unidos o tener parientes consanguíneos cerca son fuentes clave de poder para las mujeres mexicanas que emigran a ese país. De este modo, a partir de entrevistas intensivas con 26 mujeres en dos localidades, es posible generalizar sobre las influencias culturales y sociales en el comportamiento sexual y reproductivo.

En cuarto lugar, la diversidad exige poner atención a la estratificación interna de una comunidad. La meta es representar tipos de personas, no buscar una muestra matemáticamente representativa de la población. Por ejemplo, en un frasco con canicas de cinco colores, la meta sería encon trar canicas de los cinco colores para entrevistar, no descubrir qué proporción de la población es de cada color (Katz Rothman, 1986). Por supuesto, las mujeres pueden categorizarse de muchas maneras distintas, según los criterios. Lograr la diversidad se complica con el hecho de que sólo algunas categorías son visibles para alguien de afuera. Antes de comenzar el trabajo de campo, por ejemplo, una de las metas del muestreo era identificar y entrevistar a mujeres de distintas clases sociales en México. Sólo después de varios meses de trabajo de campo se volvió evidente que la ubicación exacta de la casa de los padres de la mujer en su ciudad natal (distancia y dirección respecto al zócalo) era un indicador confiable de la clase so- 
cial. De manera similar, la teoría del regateo hace hincapié en encontrar una muestra que sea diversa en términos de acceso a los recursos, aunque sólo fue en el trabajo de campo que se supo que una licencia de conducir es un sello de independencia para las mujeres migrantes.

Finalmente, el muestreo etnográfico es personal. Aquí no existe la ficción -que es una ficción en cualquier caso, incluso con los censadores- de que los contactos con los informantes existen aparte de otras relaciones sociales. Por el contrario, los contactos dependen de estas relaciones sociales, porque incluso en esta versión más sistematizada del "método de la bola de nieve" (Bernard, 1994), el investigador depende de la buena voluntad de cada informante para conocer a otros miembros de la comunidad. En el muestreo para encuestas se puede presentar un sesgo por una particular relación política entre un grupo y el cuerpo que conduce la encuesta. Un buen ejemplo de esto es el subconteo de hombres negros.jóvenes en los censos de Estados Unidos.

En la etnografia, en cambio, el peligro de sesgo se relaciona más con la personalidad y antecedentes del etnógrafo, con el tipo de gente con quien encuentra más fácil hablar. Varias inmigrantes del pueblo seleccionado, por ejemplo, habían sido de clase media ("los riquillos") en México (emigraron porque no podían pagar los préstamos del banco debido a la reciente devaluación del peso y a las transformaciones en la porcicultura). Fue más fácil entrevistarlas y tratarlas porque tendían a ser menos desconfiadas, hablaban un español más pulido y estaban más cómodas con el proceso de la entrevista. Fue importante reconocer cómo nuestros propios antecedentes de clase media podían influir en la selección de sujetos de investigación, para procurar corregir este sesgo.

\section{Demografía informal}

\section{Antecedentes de Atlanta}

El censo de 1990 reporta 108922 personas de origen hispano en Georgia (GA), de las cuales 49182 eran de origen mexicano. ${ }^{5}$ De éstas,

${ }^{5}$ U.S. Bureau of the Census, "Hispanic Origin Population by Origin for States, Counties, and Places with 100 or more Hispanics in 1990, State=Georgia", del Censo de Población y Vivienda de 1990, CP-3-3, Personas de origen hispano en Estados Unidos. 
cerca de la mitad (23 837) reside en lo que se conoce como Metro Atlanta, que abarca la ciudad de Atlanta y los condados de Clayton, Cobb, Dekalb, Fulton y Gwinett. La distribución por condado puede observarse en el cuadro 2.

CUADRO 2

Distribución de personas de origen mexicano por localidad

\begin{tabular}{lcc}
\hline Localidad & $\begin{array}{c}\text { Hispanos } \\
\text { de origen } \\
\text { mexicano }\end{array}$ & $\begin{array}{c}\text { Porcentaje del } \\
\text { total de mexicanos en } \\
z M A\end{array}$ \\
\hline Ciudad de Atlanta & 3715 & \\
Condado de Clayton & 1174 & 15.58 \\
Condado de Cobb & 3769 & 4.93 \\
Condado de Dekalb & 5708 & 15.81 \\
Condado de Fulton & 6514 & 23.95 \\
Condado de Gwinett & 3715 & 27.33 \\
Total & 23837 & 12.40 \\
& & 100.00 \\
\hline
\end{tabular}

Sin embargo, los investigadores y prestadores de servicios que trabajan con mexicanos en la zMA sugieren que esto no representa la cantidad completa. Aunque la comunidad hispánica de Atlanta no se acerca a las de California o Texas en tamaño o historia, tampoco acaba de formarse. Atlanta ha tenido una comunidad cubana desde finales de los sesenta, que aún constituye la élite de la colectividad hispana (es decir, los cuerpos directivos de la Asociación Latinoamericana y otras organizaciones hispánicas de negocios y servicio social).

La reciente oleada de inmigración mexicana a Atlanta tiene sus raíces a principios de los setenta, cuando una gran familia de un pueblo de Jalisco se mudó de Los Ángeles a Atlanta para abrir un restaurante mexicano. Han sido extremadamente exitosos, y durante los últimos 25 años ellos y sus socios han abierto más de 200 restaurantes en todo el sureste de Estados Unidos. Estos restaurantes han sido imanes para los mexicanos que provienen tanto de México como de otras ciudades de Estados Unidos, y ahora los mexicanos en Atlanta han empezado a trabajar en la construcción, procesamiento de aves de corral, fábricas de alfombras, industria eléctrica y servicio doméstico. La comunidad es lo suficientemente grande para sostener dos periódicos importantes y varios menores, así como por lo menos tres estaciones de radio y cuatro ligas de futbol. 


\section{Fase I: encontrar una comunidad}

Los equipos de futbol desempeñaron un papel clave en el plan original para encontrar un grupo de mujeres. Estos equipos generalmente son organizados por la comunidad de origen mexicano establecida en Estados Unidos, y este método ha sido utilizado exitosamente por investigadores hombres en trabajos sobre inmigrantes hombres (Massey, 1987; Cornelius, 1982; Rouse, 1991, 1992). Sin embargo, ya en el campo se volvió evidente que no se podría trabajar directamente por medio de los equipos por varias razones. Primero, las únicas "gringas" que andan solas en los partidos de futbol tratando de conocer gente son las prostitutas. Segundo, en el verano de 1995 los ataques sorpresivos del ins en toda Atlanta, incluyendo varios partidos de futbol, implicaron que las pocas mujeres que sí asistían a los partidos no aceptaran hablar con estadunidenses desconocidas (los ataques provocaron una ansiedad tan generalizada en las comunidades de inmigrantes en Atlanta, que el verano pasado muchas mujeres mexicanas sólo salían los domingos, cuando podían ir a misa y a hacer las compras acompañadas de sus esposos; esto hizo a la comunidad aún más invisible para alguien de fuera). Finalmente, las mujeres mexicanas "decentes" son en general muy amistosas si son presentadas por un conocido común, pero generalmente no hablan con extraños; varias de las que conocí en los partidos me dieron números de teléfono falsos. Así que me enfoqué, en cambio, en lo que se llama, tomándolo prestado de Corneluis (1982), el método de "la aguja en el pajar". Aquí, encontrar una comunidad equivale a buscar una aguja en un pajar, en el sentido de que para cuando se encuentra la aguja, ya se ha conocido todo lo que hay en el pajar.

Las primeras entrevistas informales tenían la intención de responder a dos preguntas: 1) ¿Cuáles son las instituciones sociales de importancia en las vidas de las mujeres mexicanas?, y 2) ¿Cuáles son sus relaciones con las personas de estas instituciones que hablan español? Los informantes para estas entrevistas exploratorias se seleccionaron de tres listas: trabajadores sociales y de salud que hablaban español, curas católicos que daban misa en español y dirigentes comunitarios, proporcionados por la embajada mexicana. En estas entrevistas se describió el proyecto como un estudio comparativo de vidas de mujeres, y se dijo que se estaba buscando un pueblo que tuviera una considerable población femenina en el área de Atlanta. Si alguien sugería un pueblo, las preguntas siguientes implicaban mayor información de- 
mográfica y económica sobre sus miembros en Atlanta, así como nombres de mujeres del pueblo. Más que tratar de entrevistar a las más de 150 personas de estas tres listas, se comenzó con las que habían sido señaladas como particularmente conocedoras de la comunidad mexicana. Las entrevistas continuaron hasta que se oía lo mismo una y otra vez (cuadro 3).

CUADRO 3

Muestreo en varias fases

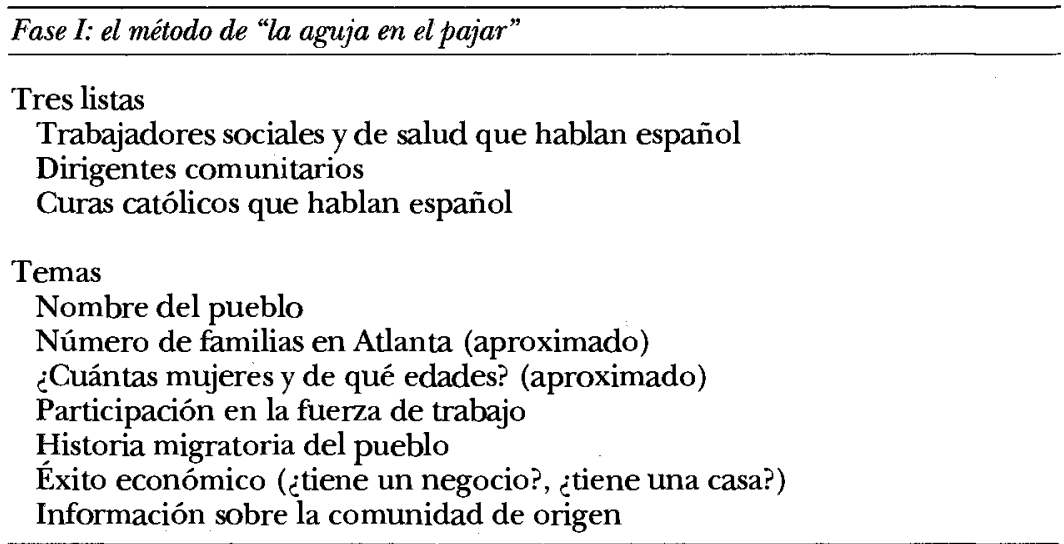

Un elemento clave de esta demografia informal es asumir una actitud crítica ante lo que la gente dice sobre la comunidad en cuestión. Con el tiempo se volvió evidente que los comentarios sobre la comunidad mexicana estaban filtrados por medio de la relación particular de las personas con dicha comunidad. Los trabajadores sociales y de salud, por ejemplo, eran en general nacidos en Estados Unidos de padres inmigrantes, y en las entrevistas se percibía una ambivalencia respecto a su identidad en relación con la gente a la que servían. Estaban políticamente comprometidos con ayudar a gente a la cual se referían como su grupo étnico, como "la raza", aunque al mismo tiempo muchos de ellos estaban individualmente comprometidos con preservar las diferencias sociales entre ellos mismos - que hablan inglés, son de clase media y nacidos en Estados Unidos-y la gente a la que sirven. Una parte de esta preservación implicaba no ir más allá de cualquier relación profesional que pudieran tener con sus clientes, en el sentido de no averiguar lo que es quizá la parte más destacada de la identidad del inmigrante: su ciudad o pueblo de origen. De ma- 
nera similar, la lista del consulado estaba comprometida con la política interna de la comunidad mexicana, y el grado en que los sacerdotes entrevistados conocían a la comunidad dependía fuertemente de su visión individual sobre el papel de la Iglesia en la acción social vs. la salvación espiritual.

Después de cuatro meses y medio, los nombres de tres pueblos habían aparecido repetidas veces. Entonces se entrevistó a varias mujeres de cada uno, para comparar qué tanto cumplían los pueblos los criterios mínimos de tener una población femenina bastante grande en Atlanta (de por lo menos 50), un flujo migratorio considerablemente establecido y cierta variación en el acceso a los recursos. Las mujeres de uno de estos sitios constantemente programaban las entrevistas cuando no estaban en sus casas, de modo que no parecieron interesadas en participar. Un segundo pueblo cumplió todos los requisitos íntegramente, aunque por razones de seguridad personal pareció mejor trabajar con otro lugar. De modo que nos decidimos por Las Mártires y el vecino rancho El Guamúchil. ${ }^{6}$ Entre los dos hay por lo menos 50 y quizás más mujeres en el área de la zMA.

El proceso seguido en la fase I fue una solución adecuada, aunque no perfecta, al problema de cómo encontrar un grupo de mujeres que vivieran en Atlanta y que fueran todas del mismo lugar. Lo ideal hubiera sido trabajar con un grupo de mujeres cuyos esposos jugaran todos en el mismo equipo de futbol organizado regionalmente (una definición local o "emic" de lo que significa ser del mismo lugar). Esto no se pudo hacer porque, como ya se dijo, no fue posible para mí como mujer trabajar por medio de los equipos de futbol. El proceso sustituto de encontrar un pueblo mediante otras redes sociales fue en todos sentidos complicado por nuestra falta de entendimiento de lo que significaba ser del mismo lugar. AI principio del trabajo de campo, por ejemplo, se preguntó a las personas de dónde eran y decían que de Guadalajara. Mucho más tarde resultó que de hecho eran de un pueblo a varias horas de Guadalajara y que ni siquiera estaba en el mismo estado. Decían que eran de Guadalajara, no por malicia o por deseos

${ }^{6}$ Estos son nombres ficticios. Si bien algunos miembros de la comunidad quieren que las publicaciones del estudio utilicen los nombres verdaderos del pueblo y el rancho, las comunidades inmigrantes en Atlanta son demasiado pequeñas para que esto sea éticamente posible. Aunque los nombres de las mujeres y algunos rasgos de identificación se cambien en todas las publicaciones basadas en esta investigación, una persona de Las Mártires o El Guamúchil que lea revistas académicas aún podría reconocer a un vecino o pariente si supiera que se trata de su pueblo. 
de esconder sus orígenes, sino porque no se había aclarado que el interés era conocer el lugar exacto de donde procedían.

Además, no fue sino hasta que estuve en México que comprendí la relación tanto geográfica como simbólica entre un rancho y el pueblo "a que pertenece". Aunque El Guamúchil, por ejemplo, está sólo a unos diez kilómetros de Las Mártires, está al otro lado del límite estatal, en Michoacán, y pertenece en realidad a una ciudad pequeña de ese estado. Como hubiera resultado evidente para un nativo del lugar, esto implica que hay una ruta de camión entre El Guamúchil y dicha ciudad, pero no entre El Guamúchil y Las Mártires. En consecuencia, la gente de El Guamúchil no va al mercado en Las Mártires, y sólo rara vez va allí para las fiestas decembrinas. Los vínculos sociales entre las dos comunidades son limitados en México, y casi inexistentes entre sus miembros residentes en Atlanta.

Para complicar las cosas aún más, una vez en Las Mártires comencé a ver que sería simplista concebir a todo el pueblo como una comunidad. Los habitantes hacen una fuerte distinción entre el pueblo en sí -las cuadras que están alrededor del zócalo y cerro abajo- y las colonias -que están cerro arriba en terrenos otorgados a la gente por el gobierno durante las últimas dos décadas. El pueblo tiene calles pavimentadas y agua entubada que llena los tanques de las casas cada tres o cuatro días. En las colonias las calles no están pavimentadas, y en las zonas donde sí hay agua en todas las casas (en las colonias más nuevas sólo hay una llave común) sólo sale una vez a la semana, y a veces con menor frecuencia. En Atlanta hay inmigrantes tanto de las colonias como del pueblo, aunque en su mayoría viven en áreas diferentes y no socializan. Cuando lo llegan a hacer, los de las colonias aún recuerdan que no hubieran osado entrar a las casas de las mujeres del pueblo, mientras que éstas no se hubieran dignado a devolver la visita. Tanto en Atlanta como en Las Mártires, es poco probable que los de la colonia conozcan a los del pueblo, y viceversa.

También fue problemático considerar a todo Atlanta como un solo lugar. Aunque a principios de los ochenta sólo había dos barrios con alta concentración de inmigrantes mexicanos, ahora están más ampliamente distribuidos. Algunos viven en barrios urbanos de unidades habitacionales y pequeños negocios propiedad de inmigrantes y con servicio de transporte público. Otros están más aislados, en sitios más rurales o en trailer parks (concentraciones de casas móviles) en los suburbios más alejados de Atlanta. Cada una de las tres localidades de México (rancho, colonias y pueblo) tiene su comunidad 
hermana equivalente en Atlanta. La gente de El Guamúchil vive casi exclusivamente en un trailer park en un suburbio a unas 25 millas al este de la ciudad. Los del pueblo de Las Mártires se han asentado en un complejo de apartamentos y un trailer park unas 20 millas al noreste de la ciudad, y los de las colonias viven en otro complejo justo al noreste de Atlanta, en un barrio que ha albergado a una fuerte comunidad mexicana (y vietnamita) durante por lo menos una década. Cada microcomunidad asiste a una iglesia católica distinta, aunque los habitantes de los suburbios han visitado en ocasiones el barrio para ir al tianguis o a uno de los muchos restaurantes mexicanos que sirven menudo los fines de semana. Así, aunque la meta era encontrar una comunidad transnacional, el resultado fue en cambio de tres comunidades transnacionales más pequeñas distribuidas en seis localidades.

Durante la recolección de la información que llevó a la selección de las comunidades de investigación, también conocí la diversidad dentro de las comunidades en sentidos que serían útiles para seleccionar a las informantes individuales en la segunda fase del muestreo. Para cuando se seleccionaron Las Mártires y El Guamúchil, se habían entrevistado suficientes mujeres para saber, por decirlo así, qué "colores de canicas" podríamos estar buscando, tanto en términos de características sociodemográficas como de las medidas de las propias mujeres en relación con el poder y la autonomía. Durante toda la fase I se preguntó sobre los factores que las mujeres consideran que las hacen más o menos independientes de sus esposos - ya que el estudio trabaja sobre el poder de regateo, es clave tener diversidad en este sentido. Las fuentes más evidentes de poder son hablar inglés y tener documentos para trabajar, aunque hay otras: saber manejar y tener coche, tener parientes consanguíneos en Atlanta y tener hijos nacidos en Estados Unidos (para poder obtener cupones de Medicaid y wic). ${ }^{7}$

\section{Fase II: elección de las informantes individuales para las historias de vida}

A medida que conocía más mujeres de la comunidad seleccionada, iba resumiendo lo aprendido sobre cada una en una tabla-rejilla (cuadro 4).

\footnotetext{
${ }^{7}$ Actualmente esto ya no es tan importante como lo fue en el momento de realizarse esta investigación, ya que los recortes en la ayuda que el gobierno de Estados Unidos proporciona a los pobres, además del creciente sentimiento anti migratorio, han convertido a los inmigrantes (tanto legales como ilegales) en sujetos no elegibles para obtener beneficios de salud y comida.
} 


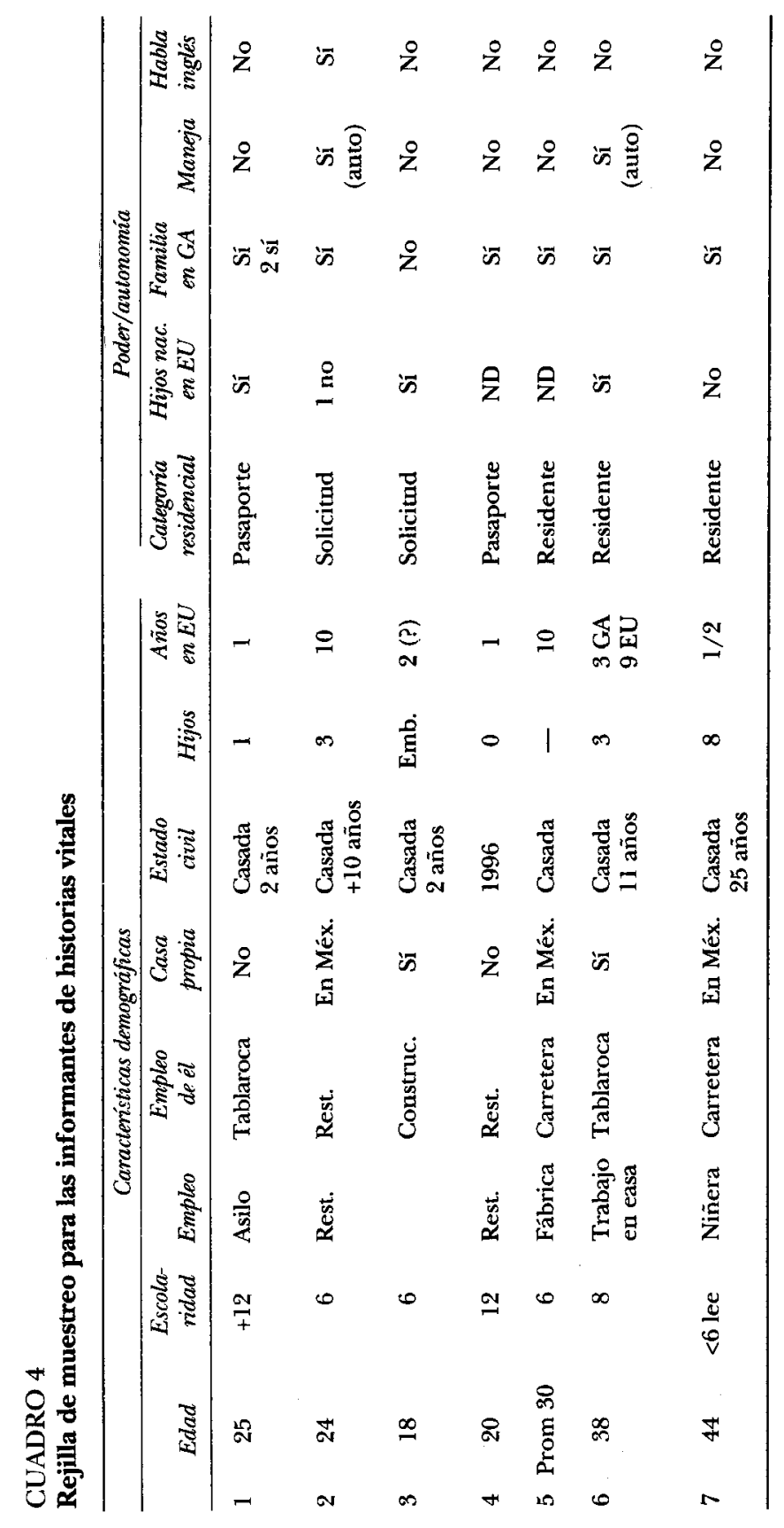




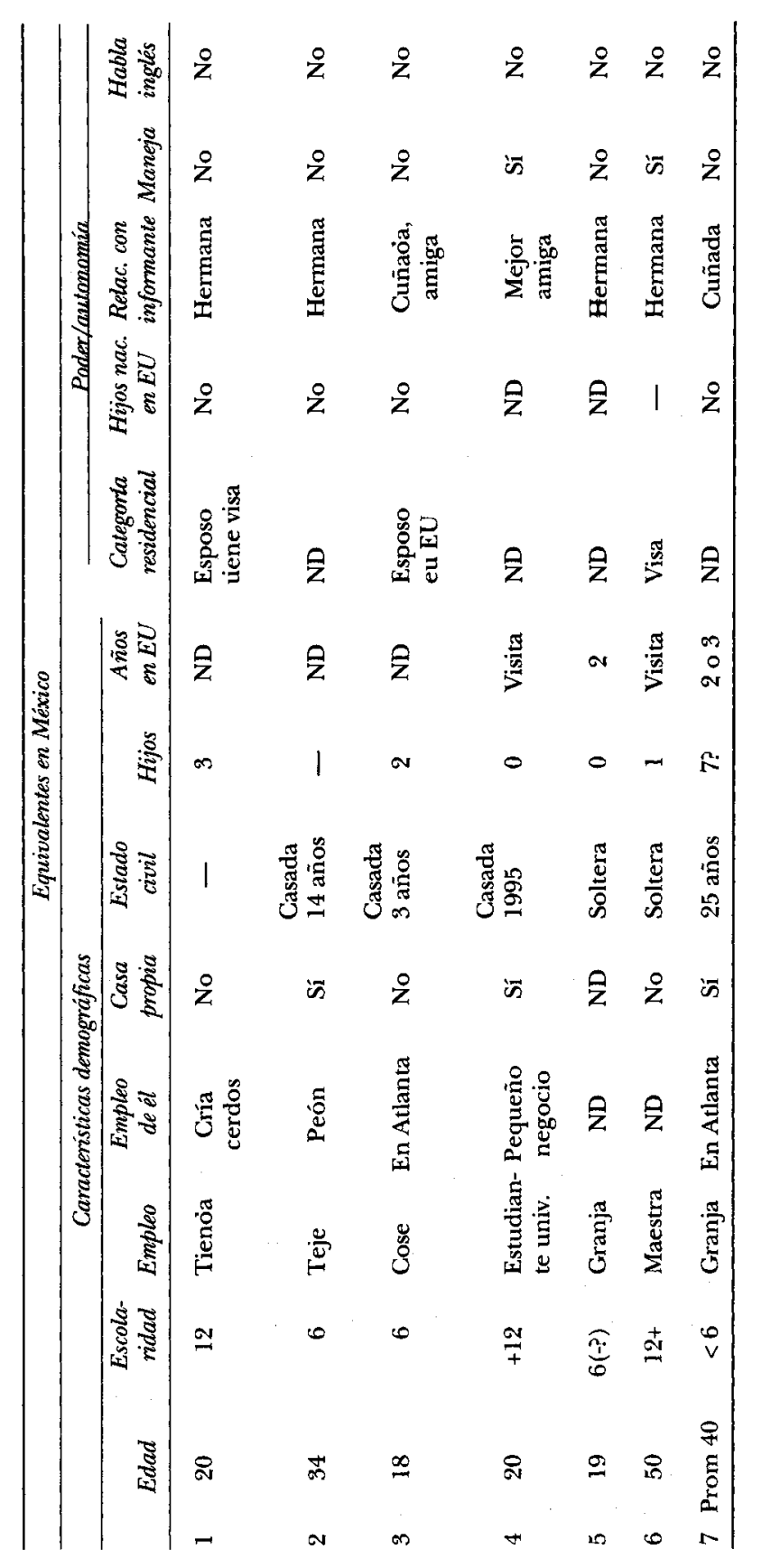


A continuación aparece la rejilla, por ejemplo, para los primeros siete pares de mujeres seleccionadas como informantes de historias de vida. La rejilla facilita la construcción de una muestra con la máxima diversidad de las características seleccionadas, pues es fácil comparar a las informantes visualmente, por ejemplo, en el número de hijos o tiempo en Estados Unidos. Así, la informante número 1 había llegado a Atlanta apenas el año anterior, con pasaporte y un título universitario, mientras que la informante 2 había estado en Estados Unidos diez años y llegó sin pasaporte ni educación preparatoria. Se eligió a las informantes una por una, tratando de maximizar la diversidad en los criterios seleccionados.

Es obvio que esto es muy distinto a la idea de usar una tabla de $2 \times 2$ con un número determinado de mujeres en cada celda para definir el tamaño de muestra; la meta es organizar y documentar el proceso de decidir a quién entrevistar. Es extremadamente flexible -el plan inicial era entrevistar a ocho informantes de historias vitales en cada localidad, aunque esto finalmente se extendió a casi el doble para crear una muestra más diversa. Las mujeres se eligieron paso a paso (y no todas a la vez) a medida que se aprendía más sobre la comunidad y que se conseguían más contactos.

La siguiente etapa en el muestreo es "aparear" a las mujeres con amigas o parientes en México. Desde el principio las mujeres sabían que iría a sus lugares de origen a entrevistar a sus familias, y a medida que se progresaba hacia el final de las seis entrevistas de historias de vida se les pidió que recomendaran a una hermana, cuñada, prima o amiga que fuera muy parecida a ellas pero que estuviera todavía en la localidad de origen.

Esta estrategia sirvió para varios propósitos. Primero, aseguró cierta eficiencia en la mitad mexicana del trabajo de campo, porque llegé al lugar con contactos que ya me estaban esperando y que estaban de hecho ansiosas por conocerme, en lugar de tener que comenzar de cero. Lo más importante es que aseguró cierta semejanza socioeconómica entre los dos grupos de mujeres entrevistadas. Como las mujeres que emigran son sin duda diferentes en varios aspectos importantes de las que no lo hacen, este apareamiento por lo menos logró cierta similitud en las circunstancias sociales y económicas de ambas. Además, la similitud estructural de los dos grupos permite una exploración más profunda de otros aspectos de la selectividad migratoria, como el papel de las metas personales, las relaciones maritales y de otro tipo y las etapas de formación familiar. 


\section{Conclusión}

El muestreo etnográfico sistemático es un primer paso importante en la construcción de una etnografía que pueda comunicar resultados generalizables a elaboradores de políticas y académicos entrenados en las convenciones de un muestreo demográfico más tradicional (Holland et al., 1992; Johannsen, 1992; Sanjek, 1991). Es especialmente importante en la investigación sobre sexualidad, donde la atención (por lo menos en el mundo occidental) se ha centrado en pequeños grupos urbanos cuya sexualidad es "visible" porque se percibe como problemática -por ejemplo, homosexuales y prostitutas. Ha sido importante explorar estas sexualidades marginales tanto por su propio derecho como por lo que pueden enseñar sobre la opresión y resistencia en la construcción social de la sexualidad. No obstante, si la investigación etnográfica sobre sexualidad ha de voltear la mirada hacia objetos (o sujetos) menos obvios en las sociedades complejas, será cada vez más importante tratar con cuestiones de generalización. A partir de este estudio no se podrá concluir qué porcentaje de mujeres migrantes es más probable que presente ciertas conductas de protección, o con qué se correlacionan dichas conductas en algún sentido estadístico, aunque sí se podrá concluir cómo se construyen socialmente la sexualidad y el género en esta comunidad inmigrante, y cómo dentro de esta construcción social ciertos factores ofrecen a las mujeres mayor o menor poder en sus relaciones sexuales. La generalización etnográfica se centra en lo que significan para la gente las conductas, no en el porcentaje de la población que las realiza. No es lo mismo que la generalización estadística, aunque finalmente el sentido de la investigación interdisciplinaria no es que todas las disciplinas hagan lo mismo, sino que cada una aporte lo que hace mejor.

\section{Bibliografía}

Abu-Lughod, L. (1993), Writing Women's Worlds: Bedouin Stories, Berkeley, University of California Press.

Alarcón, R. (1988), "El proceso de norteñización: impacto de la migración internacional en Chavinda, Michoacán", en Thomas Calvo y Gustavo López (eds.), Movimiento de población en el occidente de México, Zamora, El Colegio de Michoacán, pp. 337-357.

Anzaldua, G. (1987), Borderlands/La Frontera: The New Mestiza, San Francisco, Spinsters/Aunt Lute Publishers. 
Asad, T. (1994), "Ethnographic Representation, Statistics, and Modern Power", Social Research: An International Quarterby of the Social Sciences, vol. 61, núm. 1, pp. 55-88.

Bernard, H.R. (1994), Research Methods in Cultural Antrophology: Qualitative and Quantitative Approaches, Newbury Park, Sage.

Bronfman, M. y H. Gómez Dantes (s.f.), "Sexualidad, enfermedades de transmisión sexual y salud reproductiva: el oscuro objeto del deseo", México, El Colegio de México (mimeo.).

Cornelius, W. A. (1982), "Interviewing Undocumented Immigrants: Methodological Reflections Based on Fieldwork in Mexico and the U.S.", International Migration Review, vol. 16, pp. 378-411.

Cornwall, A. (1992), "Body Mapping in Health RRA/PRA", RRA Notes, núm. 16 (edición especial sobre aplicaciones a la salud), Londres, International Institute for Environment and Development, Sustainable Agriculture Program.

Fernández, C. (1988), "Migración hacia los Estados Unidos: caso Santa Inés, Michoacán", enThomas Calvo y Gustavo López Castro (eds.), Migración en el occidente de México, Zamora, El Colegio de Michoacán, pp. 113-124.

Gulati, L. (1981), Profiles in Female Poverty, Nueva Delhi, Hindustan Publishing Company.

Holland, J. et al. (1992), "Risk, Power, and the Possibility of Pleasure: Young Women and Safer Sex", AISA Care, vol. 4, núm. 3, pp. 273-283.

Hondagneu-Sotelo, P. (1994), Gendered Transitions: Mexican Experiences on Immigration, Berkeley, University of California Press.

Johannsen, A. M. (1992), "Applied Anthropology and Post-Modernist Ethnography", Human Onganization, vol. 51, núm. 1, pp. 71-81.

Kahn, J. R. (1988), "Immigrant Selectivity and Fertility Adaptation in the United States", Social Forces, vol. 67, núm. 1, pp. 108-128.

Katz Rothman, B. (1986), The Tentative Pregnancy: Prenatal Diagnosis and the Future of Motherhood, Nueva York, Penguin Books.

Kearney, Michael (1986), "From Invisible Hand to Visible Feet: Antropological Studies of Migration and Development", Annual Reviews in Anthropology, vol. 15, pp. 331-361.

Massey, D. S. (1987), "The Ethnosurvey in Theory and Practice", International Migration Review, vol. 21, núm. 4, pp. 1498-1522.

— , R. Alarcón, J. Durand y González (1987), Retum to Aztlán: The Social Process of International Migration from Western Mexico, Berkeley, University of California Press.

— J. Arango, G. Hugo, A. Kouaouci, A. Pellegrino y J. E. Taylor (1993), "Theories of International Migration: A Review and Appraisal", Population and Development Review, vol. 19, núm. 1, pp. 431-466.

Mintz, S. (1960), Worker in the Cane: A Puerto Rican Life Histony, Nueva York, Norton.

Mummert, G. (1992), "Reshaping of Gender and Generational Relations Among Rural Mexican Migrants to the U.S.”, trabajo presentado en el 
XVII Congreso Internacional de la Latin American Studies Association, Los Ángeles, 24-27 de septiembre.

Rouse, R. (1991), "Mexican Migration and the Social Space of Postmodernism", Diaspora, vol. 1, núm. 1, pp. 8-23.

(1992), "Making Sense of Settlement: Class Transformation, Cultural Struggle, and Transnationalism among Mexican Migrants in the United States", en N. Glick Schiller, L. Basch y C. Blanc-Szanton (eds.), Towards a Transnational Perspective on Migration, Nueva York, New York Academy of Sciences (Annals of the New York Academy of Sciences, 645), pp. 25-52.

Sanjek, R. (1991), "The Ethnographic Present", Man, núm. 26, pp. 609-628.

Trigueros, P. y J. Rodríguez Piña (1988), "Migración y vida familiar en Michoacán", en Thomas Calvo y Gustavo López Castro (eds.), Migración el en el occidente de México, Zamora, El Colegio de Michoacán, pp. 201-221.

Vance, C. (1991), "Anthropology Rediscovers Sexuality: A Theoretical Comment", Social Science and Medicine, vol. 33, núm. 8, pp. 875-884. 
